\section{In and out}

Nuclear import and export signals can be hard to detect in protein sequences because of their small size and lack of coherent motifs. In this issue, Rhee et al present a genetic method for detecting these signals based on a functional assay in yeast cells. First they modified the bacterial LexA protein, to abolish its intrinsic nuclear targeting activity, and then fused it to a sequence the yeast GAL4 activation domain, and the protein to be tested. If the cell contains a functional nuclear import signal, the fusion protein will enter the cell nucleus, where and activate the expression of $\beta$-galactosidase and a histidine biosynthesis gene. To assay for nuclear export, they made another construct in which fusion to proteins that contain a nuclear export signal will redirect the protein into the cytoplasm, reducing $\beta$-galactosidase expression and growth on histidine deficient media (see p. 433).

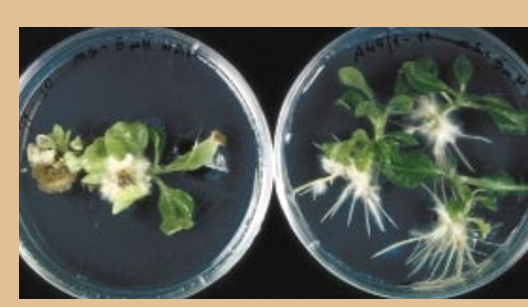

When introducing foreign genes into plants, scientists must also introduce a resistance gene to herbicide or antibiotics-this allows the cells with the incorporated transgenes to be regenerated on selectable media. However, while it has not ben demonstrated that marker genes can "escape" to weedy relatives or gut bacteria, their safety has nonetheless become a flash point in the debate over safety of GM foods. Since marker genes are only needed during the plant transformation process, it should be possible to remove marker genes from crops after the transgenic material has been selected, which would improve the public acceptance of genetically engineered crops. On page 442, Meyer and colleagues describe a vector that mediates deletion of marker genes from transgenic tobacco. They placed the marker gene, which confers resistance to kanamycin, between bacteriophage lambda sequences called Attachment $\mathbf{P}$ regions, which mediate intrachromosomal recombination. They also included a gene called tms2, which inhibits growth in the presence of certain hormones, and therefore can be used to select transformants in which the marker gene was deleted through intrachromosomal recombination.

This Month in Nature Biotechnology written by Natalie DeWitt and Robert Frederickson.

Pluripotent immortal stem cell lines have become an important new tool to study early human development and also possess great clinical potential for transplantation and tissue regeneration therapies. In this issue, Pera and colleagues have confirmed the generation of embryonic stem (ES) cells from human blastocysts and have demonstrated their subsequent differentiation into a neuronal progenitor cell in vitro. Not only do these workers verify the characteristics of human

ES cells, they substantiate the differences and similarities

between other types of pluripotent cells. They also

demonstrate expression of Oct $3 / 4$, which should

allow parallels to be drawn with murine ES cells

which have been more extensively studied to date for

practical reasons (see p. 399).

The infusion of large numbers of antigenspecific cytotoxic T lymphocytes (CTLs) is a promising therapeutic strategy in the fight against certain cancers and infectious diseases. This process currently involves the time-consuming differentiation or stimulation of natural antigen-presenting dendritic cells from the bone marrow or peripheral blood, respectively, of individual patients. These cells must then be pulsed with antigens to condition them to induce antigen-specific cytotoxic $\mathrm{T}$ cells in vitro. In this issue, Latouche and Sadelain (p. 405) describe an alternative method: the use of stable artificial antigen presenting cells (AAPC). They derived xenogeneic murine fibroblasts expressing specific human HLA class-I peptide complexes along with CD80, CD54, and CD58 by retroviral transduction. These cells could be efficiently expanded in vitro and used to stimulate $\mathrm{T}$ cells of any patient of a given human leukocyte antigen (HLA) type. These workers demonstrated potent induction and expansion of CTLs in the context of HLA type A2.1, which is present in $40 \%$ of the Caucasian population.

\section{Microarrays get into print}

One way of fabricating DNA microarrays is to spot pre-synthesized oligonucleotides directly onto a glass plate. In this issue (p. 438), Okamoto et al. improve on this method using a miniaturized "Bubble Jet" device to spot the oligonucleotides. Their technology ejects picoliter sized droplets onto the glass surface, with lower cost and greater uniformity than existing microspotting technologies. They further enhanced the robustness of the microarrays by spotting onto a glass surface coated with bifunctional crosslinkers that covalently bind to a thiol group at one end of the oligonucleotides probes. They demonstrated that microarrays fashioned by this method could be used to distinguish single base mismatches from perfect matches after probing with synthetic oligonucleotides or PCR amplified fragments.

\section{A "cool" new system for gene expression}

Heterologous protein expression is useful for the production of recombinant proteins for both therapeutic and basic research purposes. Regulated expression systems can be useful for maximizing production levels without inhibiting cell proliferation, particularly if the protein is toxic to the cell. Inducible expression systems are usually regulated at the level of transcription by inducer or repressor molecules, and often suffer from leaky background expression and/or poor inducibility. In this issue, Boorsma et al. (p. 429) describe a new system, based on transcription of a heterologous gene by a modified temperaturesensitive RNA-dependant RNA replicase from alphavirus. Their modified viral replicase is only active at temperatures below $35^{\circ} \mathrm{C}$, and exhibits low background and high inducibility of various reporter genes in both transiently and stably transfected cells.

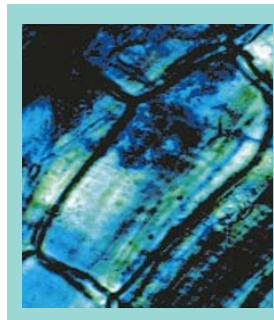

Phosphate-a limiting nutrient for agriculture-is applied to soils worldwide by the millions of tons. However, in alkaline or acid soils, phosphate forms insoluble precipitates making most of it unusable to plants. Phosphate fertilizer run off is a major environmental pollutant, and improving the ability of plants to absorb and use phosphate has become a key concern. In this issue, Herrera-Estrella and colleagues (p. 450) tackle this problem by engineering plants that require less phosphate to achieve optimal growth in alkaline soil. Acid applied to soil was previously known to free up phosphate in insoluble calcium phosphate complexes. They overexpress citrate synthase from Pseudomonas in tobacco, making plants that secrete citric acid into the soil, converting the insoluble complexes into a usable form. 